\title{
Child psychiatry and child sexual abuse
}

In the light of new contexts, a working group of the Child and Adolescent Specialist Section of the College has produced a comprehensive new document reviewing the current role of child and adolescent psychiatry in the field of child sexual abuse. There is also mention of the role of general and forensic psychiatry. The need for this review has arisen due to major changes in health and personal social service provision, the law, developments in clinical practice and new research findings.

The changes outlined in the document include The Children Act (1989), new rules for receiving children's video-taped evidence in criminal proceedings, and the subsequent publication of the Memorandum of Good Practice in Interviewing Children. Increasing recognition of a variety of issues is considered, including the importance of culture, ethnicity and disability; the abuse of boys; abuse by adolescents and inappropriate or coercive sexual involvement of children by other children; sexual abuse by women; abuse within institutions and by alternative carers; organised abuse within ritualised and religious practice.

The document discusses the various types of work in which child psychiatric teams are appropriately involved and are offering a major contribution in the field. As well as providing post-protection therapy, these include consultation at various stages of the process of professional intervention, training, research and contributing a developmental and child mental health perspective to the wider professional network. There is a recognition that child sexual abuse issues arise in diverse settings and clinical situations.

The specific role of child psychiatrists in interviewing children is considered, with particular emphasis on assessment as opposed to investigative interviews. The nature of forensic civil and criminal work is discussed, as are second opinions and claims from the Criminal Injuries Compensation Board which are being increasingly made on behalf of children who have been sexually abused. Child psychiatrists are not infrequently requested to contribute to applications and appeals. Issues of confidentiality and consent which have given rise to recent problems are examined.

The document is richly referenced, the latter highlighting recent publications of research findings and innovative practice.

Bloomfield Clinic

Guy's Hospital

London SEI 9RT

The full report will be available from the College Publications Department.

\section{GMC revised guidance on confidentiality and child abuse}

At the May 1993 meeting of the General Medical Council there was agreement to amend paragraph 83 of the booklet Professional Conduct and Discipline: Fitness to Practice (January 1993) as follows:

"Deciding whether or not to disclose information is particularly difficult in cases where a patient cannot be judged capable of giving or withholding consent to disclosure. One such situation may arise where a doctor believes that a patient may be the victim of abuse or neglect. In such circumstances the patient's interests are paramount and will usually require the doctor to disclose information to an appropriate, responsible person or an officer of a statutory agency".

The Council also agreed to amend paragraph 86 of the guidance dealing with disclosures in the public interest to read:

"Rarely, cases may arise in which disclosure in the public interest or in the interests of an individual, may be justified, for example, a situation in which the failure to disclose appropriate information would expose the patient, or someone else, to risk of death or serious harm".

\section{Academic psychiatry post in Malawi}

The Overseas Liaison Committee of the College has been asked to invite interested consultants to apply for an academic psychiatry post in Malawi. For further details of this post please contact $\mathrm{Dr}$ Maureen Wilkinson, Orchard House, 59a Chilton House, Long Crendon, Bucks. HP18 9BZ. 